\title{
A nationwide utilization survey of therapeutic drug monitoring for five antibiotics in South Korea
}

This article was published in the following Dove Press journal:

Infection and Drug Resistance

\author{
Rihwa Choi ${ }^{1,2}$ \\ Hye In Woo' \\ Hyung-Doo Park' \\ Soo-Youn Lee ${ }^{1,3}$ \\ 'Department of Laboratory Medicine and \\ Genetics, Samsung Medical Center, \\ Sungkyunkwan University School of \\ Medicine, Seoul, Republic of Korea; \\ ${ }^{2}$ Department of Laboratory Medicine, \\ Green Cross Laboratories, Yongin, \\ Gyeonggi, Republic of Korea; \\ ${ }^{3}$ Department of Clinical Pharmacology \\ and Therapeutics, Samsung Medical \\ Center, Sungkyunkwan University School \\ of Medicine, Seoul, Republic of Korea
}

Purpose: The current status of therapeutic drug monitoring (TDM) assay utilization by clinical laboratories in South Korea remains little known. We investigated the TDM status of five antibiotics known for nephrotoxicity (vancomycin, amikacin, gentamicin, tobramycin, and teicoplanin) for the improvement of TDM in South Korea among patients with infectious diseases using a cross-sectional nationwide survey.

Patients and methods: We developed an online questionnaire and collected responses using a user-friendly web-based platform. The survey included questions about laboratory characteristics, implementation and operation of drug assays, implementation and operation of TDM consulting services, patient needs, and barriers to providing better TDM service including expectations and concerns about other platform-based drug assays.

Results: Among a total of 235 clinical laboratories, 112 (47.7\%) responded, and 62 of the responding laboratories (55.4\%) possessed drug assay facilities. Only $41.2 \%$ to $58.1 \%$ of respondents were providing TDM consulting services for each antibiotic. Respondents indicated that there are unmet needs regarding drug assays and TDM consultation as well as barriers to TDM utilization including high operating costs, lack of knowledge about TDM, lack of user-friendly software, lack of medical and laboratory information systems that can access patient information critical for TDM dose calculation, and reimbursement issues.

Conclusion: This study, the first nationwide survey addressing these questions, showed that there are barriers against the utilization of TDM in South Korea. These barriers may be addressed by improving drug assays and TDM consulting services with the goals of new analytical method development, better interpretation of results, consultation services, and quality control.

Keywords: questionnaire, TDM service, TDM consulting, point-of-care

\section{Introduction}

Aminoglycosides are antibiotics that are generally active against many aerobic Gram-negative bacteria and some aerobic Gram-positive bacteria, and are principally used to treat serious infections including drug resistant tuberculosis and certain nontuberculous mycobacterial infections (in combination with other antimycobacterial agents). ${ }^{1}$ Based on the Global Antimicrobial Resistance Surveillance System Report 2016-2017 of the World Health Organization (WHO), among patients with suspected bloodstream infections, the proportion with bacteria resistant to at least one of the most commonly used antibiotics varied widely between countries, ranging from 0 to $82 \% .^{2}$ Vancomycin, a type of glycopeptide antibiotic, as well as systemically administered aminoglycosides (eg, amikacin, gentamicin, tobramycin) are widely used for the treatment of infectious disease and the most
Correspondence: Soo-Youn Lee Department of Laboratory Medicine and Genetics, Department of Clinical Pharmacology and Therapeutics, Samsung Medical Center, Sungkyunkwan University School of Medicine, 8I Irwon-ro,

Gangnam-gu, Seoul 0635I, Republic of Korea

Tel +8 2234101834

Fax +8 2234102719

Email suddenbz@skku.edu 
frequently monitored antibiotics because of their narrow therapeutic indexes and potential adverse effects. ${ }^{3}$ Therapeutic Drug Monitoring (TDM) is an evolving tool used to optimize the administration of antimicrobial agents that can significantly increase clinical efficacy and decrease toxicity in patients treated with antibiotics. ${ }^{4-6}$ Nephrotoxicity has been reported as ranging from as low as $0 \%$ in the absence of concurrent nephrotoxins to over $40 \%$ in patients treated with vancomycin. ${ }^{7,8}$ As many as $20 \%$ of patients treated with aminoglycosides experience ototoxicity and acute kidney injury due to acute tubular necrosis, a relatively common complication of aminoglycoside therapy, defined as a rise in the serum creatinine concentration of more than $0.5-1 \mathrm{mg} / \mathrm{dL}$ ( 44 to $88 \mathrm{umol} / \mathrm{L}$ ) or a 50 percent increase in serum creatinine concentration from baseline. ${ }^{9-11}$ The use of glycopeptides has rapidly increased in the past 10 years because of the exponential spread of methicillin-resistant Staphylococcus aureus (MRSA), while teicoplanin, another glycopeptide antibiotic, has become increasingly popular. ${ }^{12}$ The role of TDM in teicoplanin use is primarily to ensure treatment efficacy. ${ }^{12}$

TDM service could effectively mediate between laboratories and clinicians to ensure appropriate adjustments in drug regimens with a system for improving the utilization of TDM being official report forms including the drug concentrations found, the desired therapeutic concentration range, and recommendations to achieve the latter. ${ }^{4}$ TDM relies on the rapid and accurate determination of drug levels in patients with dose adjustments recommended if these are not consistent with expected, or target, concentration ranges. ${ }^{3,4}$ In this context, TDM drug assays and consultations provided by professionals are important components of reliable TDM. ${ }^{4}$

TDM-based therapeutic approaches for these antibiotics are still not widely utilized in routine clinical practice. ${ }^{13,14}$ Although it is hypothesized that the high cost of some of the assays may discourage providers, there are no data to explain why TDM is not used more. ${ }^{14}$ There have been no studies of nationwide TDM practices including both drug assays and TDM consultation services. Such studies could help improve the quality of the health care system in South Korea. In this study, we surveyed TDM utilization among clinical laboratories in South Korea, including drug assays and TDM consulting services, associated with five antibiotics (vancomycin, amikacin, gentamicin, tobramycin, and teicoplanin). Our goal was to determine the current state of utilization, and to identify areas for improvement. We also aimed to investigate barriers impeding the utilization of drug assays and TDM consulting services, and to survey expectations and concerns about new drug assays and TDM consulting services, to formulate strategies for the improvement of health care provision by medical specialists and clinical laboratories.

\section{Materials and methods Type of study}

We conducted a nationwide cross-sectional survey that used an online questionnaire to assess practices for five antibiotics (amikacin, gentamicin, tobramycin, vancomycin, and teicoplanin), including drug assays and TDM consultation services in South Korea.

\section{Study population}

We aimed to survey all South Korean clinical laboratory medical professionals in laboratory medicine (medical doctors), in both private and public hospitals, in local and tertiary hospitals and in specialized referral laboratories. In the absence of an exhaustive national list of such professionals, we contacted medical professionals on the email list of the Korean Society for Laboratory Medicine.

\section{Study procedures}

The survey took place from March 20 to March 27, 2018. Invitations to participate were emailed to 235 members of the Korean Society for Laboratory Medicine (KSLM) who were 235 medical directors or medical doctors in laboratory medicine (including clinical pathologists whose medical board certification is laboratory medicine) who work in clinical laboratories in South Korea.

\section{Ethical approval and informed consent}

The research related to human use has been complied with all the relevant national regulations, institutional policies and in accordance the tenets of the Helsinki Declaration, and has been approved by the Institutional Review Board of Samsung Medical Center (SMC 2017-12-038-002). Written informed consent was obtained from all survey participants (by signing the consent form).

\section{Questionnaire development}

We developed the questionnaire based on previous studies and guidelines regarding TDM services ${ }^{14-20}$ and collected responses using a user-friendly web-based platform. Data collection through online surveys is an efficient means to 
collect and analyze data. We tested the questionnaire in a volunteer group of six medical directors at six different clinical laboratories before it was made available for general responses.

\section{Data collection}

The questionnaire focused on five antibiotics, vancomycin, amikacin, gentamicin, tobramycin, and teicoplanin, and sought to identify the following information: general information about clinical laboratories, current status regarding operating drug assays and TDM consulting services for each antibiotic, and barriers to and needs for new drug assays and TDM consulting services for improvement. A condensed version of the questionnaire is provided in Table 1. The questionnaire also included questions about expectations regarding point-of-care tests or smaller scale platforms for drug assays. It consisted of multiple choice and open-ended questions.

\section{Statistical analyses}

Data were analyzed using Microsoft Excel 2013. Descriptive statistics with frequencies were used when appropriate. Percentages were calculated based on the number of respondents who answered each question.

\section{Results}

\section{Current status of drug assays for TDM services}

Among a total of 235 clinical laboratories, 112 (47.7\%) responded to any questionnaires. Among the 112 responding laboratories, 62 (55.4\%) provide TDM service based on their own instrument results for quantification analyses of any one of the five antibiotics, and 50 provide TDM service based on results from referral laboratories. The characteristics of the
112 responding laboratories are summarized in Table 2. Most respondents that did not perform drug assays were clinical laboratories in secondary hospitals $(44 / 50,88.0 \%)$. All of the clinical laboratories without drug assays had $<1,000$ hospital beds, and $98.0 \%(49 / 50)$ of these clinical laboratories had $\leq 50$ medical technologists.

The current status of drug assays performed in 62 clinical laboratories that had in-house drug assay facilities was investigated through questionnaires and the results are summarized in Figure 1 and Table 3. Among the 62 clinical laboratories with in-house drug assays, less than 10 were providing antibiotic analysis for amikacin, gentamicin, tobramycin, and teicoplanin. Therefore, only $8.9 \%$ of all clinical laboratories in South Korea perform drug assays for those antibiotics. Most laboratories with in-house drug assays were performing assays $\geq 5$ times a week. In most laboratories, commercialized quality control (QC) materials used at least two concentrations for drug assays to assess internal QC, except for teicoplanin. For external quality assurance (EQA), 20.0-28.6\% of respondents were not participating in proficiency testing according to the survey data provided by Korean Association of External Quality Assessment Service (K-EQAS) for antibiotics other than vancomycin (K-EQAS does not perform proficiency testing surveys for teicoplanin).

Among clinical laboratories providing drug assay results to physicians, the vast majority (92.5-95.8\%) use referral laboratories for all antibiotics except for vancomycin. The current status of drug assay result reporting and TDM consulting services for the clinical laboratories we surveyed are summarized in Table 4. The average number of drug assay requests by physicians per month for most laboratories was $<50$ except for vancomycin, and the number of requests for TDM consulting services was $\leq 10$ per month for most laboratories except for

Table I Aspects included in the TDM survey of clinical laboratories in South Korea

\begin{tabular}{|l|l|}
\hline Aspects & Question Items \\
\hline $\begin{array}{l}\text { Baseline characteristics of clinical } \\
\text { laboratories }\end{array}$ & Types of clinical laboratories, numbers of hospital beds, and numbers of medical technologists \\
\hline Implementation of drug assays & $\begin{array}{l}\text { Test items, operating hours and frequency, monthly workload (number of test requests), turnaround times, } \\
\text { internal quality control, and external quality assurance }\end{array}$ \\
\hline $\begin{array}{l}\text { Implementation of TDM consult- } \\
\text { ing service }\end{array}$ & $\begin{array}{l}\text { TDM consulting service provider (department doing TDM consulting), monthly workload (number of } \\
\text { requests), turnaround times, and software for dosage calculation }\end{array}$ \\
\hline $\begin{array}{l}\text { Needs for other platform-based } \\
\text { drug assays }\end{array}$ & $\begin{array}{l}\text { Needs for point-of-care drug assays, needs for smaller scale platform drug assays, reasons for the needs for } \\
\text { other platform-based drug assays, requirements, expectations and concerns regarding other platform-based } \\
\text { drug assays, considerations or intentions to implement and/or use other platform-based drug assays }\end{array}$ \\
\hline
\end{tabular}

Abbreviation: TDM, therapeutic drug monitoring. 
Table 2 Characteristics of 112 clinical laboratories in South Korea

\begin{tabular}{|c|c|c|c|c|}
\hline \multirow[t]{2}{*}{ Laboratory characteristics } & \multicolumn{2}{|c|}{$\begin{array}{l}\text { Laboratories } \\
\text { with in-house } \\
\text { drug assays } \\
(n=62)\end{array}$} & \multicolumn{2}{|c|}{$\begin{array}{l}\text { Laboratories } \\
\text { without in- } \\
\text { house drug } \\
\text { assays }(n=50)\end{array}$} \\
\hline & $\mathbf{n}$ & $\%$ & $\mathbf{n}$ & $\%$ \\
\hline \multicolumn{5}{|l|}{ Type of clinical laboratories } \\
\hline $\begin{array}{l}\text { Secondary hospitals } \\
\text { Tertiary hospitals } \\
\text { Referral laboratory }\end{array}$ & $\begin{array}{l}25 \\
32 \\
5\end{array}$ & $\begin{array}{l}40.3 \\
51.6 \\
8.1\end{array}$ & $\begin{array}{l}44 \\
4 \\
2\end{array}$ & $\begin{array}{l}88.0 \\
8.0 \\
4.0\end{array}$ \\
\hline \multicolumn{5}{|l|}{$\mathbf{N}$ of hospital beds } \\
\hline $\begin{array}{l}<300 \\
300-499 \\
500-999 \\
1,000-1,499 \\
1,500-1,999 \\
>2,000 \\
\text { None (referral laboratory) }\end{array}$ & $\begin{array}{l}1 \\
2 \\
36 \\
12 \\
3 \\
3 \\
5\end{array}$ & $\begin{array}{l}1.6 \\
3.2 \\
58.1 \\
19.4 \\
4.8 \\
4.8 \\
8.1\end{array}$ & $\begin{array}{l}23 \\
13 \\
18 \\
0 \\
0 \\
0 \\
2\end{array}$ & $\begin{array}{l}41.1 \\
23.2 \\
32.1 \\
0.0 \\
0.0 \\
0.0 \\
3.6\end{array}$ \\
\hline \multicolumn{5}{|l|}{$\mathbf{N}$ of medical technologists } \\
\hline $\begin{array}{l}I-20 \\
2 I-50 \\
5 I-100 \\
100-150 \\
>150\end{array}$ & $\begin{array}{l}4 \\
40 \\
11 \\
2 \\
5\end{array}$ & $\begin{array}{l}6.5 \\
64.5 \\
17.7 \\
3.2 \\
8.1\end{array}$ & $\begin{array}{l}35 \\
14 \\
1 \\
0 \\
0\end{array}$ & $\begin{array}{l}70.0 \\
28.0 \\
2.0 \\
0.0 \\
0.0\end{array}$ \\
\hline
\end{tabular}

Abbreviation: TDM, therapeutic drug monitoring.

vancomycin. Turnaround times for drug assays were $\geq 24 \mathrm{hrs}$ in most laboratories except for vancomycin. However, among laboratories providing TDM consulting services, turnaround time was 4-24 hrs in most laboratories. Multiple departments in these hospitals/laboratories were providing TDM consulting services using different computer software for antibiotic dose calculation. Clinical laboratories providing drug assay results to physicians indicated that they provide various ranges of target concentrations for TDM of each antibiotic and that they were not providing target concentrations with drug assay results (Figure 2). Several reasons were given by clinical laboratories for using referral laboratories or not providing drug assay results, and the most common was low number of physician requests followed by high operating costs.

\section{Current status and barriers to TDM consultation service provision}

Meanwhile, for TDM consultation services, 51.9\% (27/52) of laboratories without in-house drug assays responded that they would not provide TDM consultation services even if they performed and or could perform in-house drug assays. The most common reason for not providing/would not provide TDM consulting services to physicians was a lack of medical professionals available for TDM consultation, followed by difficulties obtaining clinical information necessary for TDM consultation, such as time the blood sample was taken, time dosage was received, dose, duration, dosage form, patient demographics, co-medications, and indication for monitoring 4. Other responses to open ended questions exploring reasons for providing drug assays and TDM consultation services were lack of middle wares interfacing between medical and laboratory information systems and dosage calculation software, lack of standardized guidelines for TDM, and the current Korean reimbursement system, which uses Refined DiagnosisRelated Groups and may decrease TDM utilization.

\section{Expectations for other platform-based drug assays such as point-of-care or smaller scale devices}

Among 112 respondents, 47 (42.0\%) clinical laboratories responded that there were needs for other platform-based drug assays, such as point-of-care (POC) assays, and 66 $(58.9 \%)$ clinical laboratories responded that smaller-scale platform devices are needed (Figure 3). Among those respondents, laboratories with in-house drug assays also indicated that they could consider implementing the smaller-scale platform devices in their laboratories, and laboratories without in-house drug assays responded that they would consider replacing their current referral processes and stop utilizing other laboratories for drug assays. A number of respondents considered operating smallerscale devices to be similar to operating the main instruments for drug assays that they were currently using. The most common reason given for desiring smaller-scale devices to perform other platform-based drug assays was cost-effectiveness, while for POC devices, the most common need indicated for other platforms was short turnaround time.

Our questionnaire included queries about expectations and concerns regarding requirements for new and other platform-based drug assays (Figure 4). Laboratories with in-house drug assays answered that agreement between test results and results obtained using comparative instruments was the most important requirement. Regarding concerns about other platform-based devices, more than 


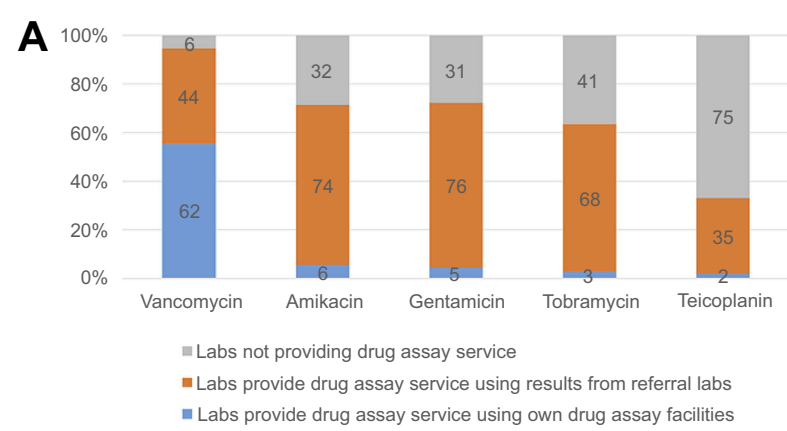

B

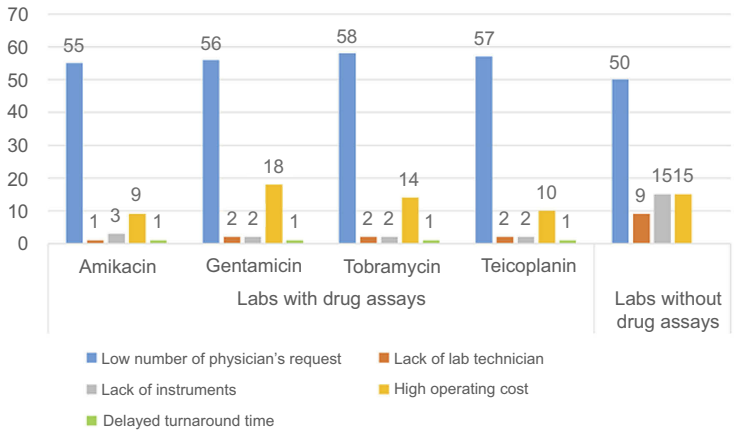

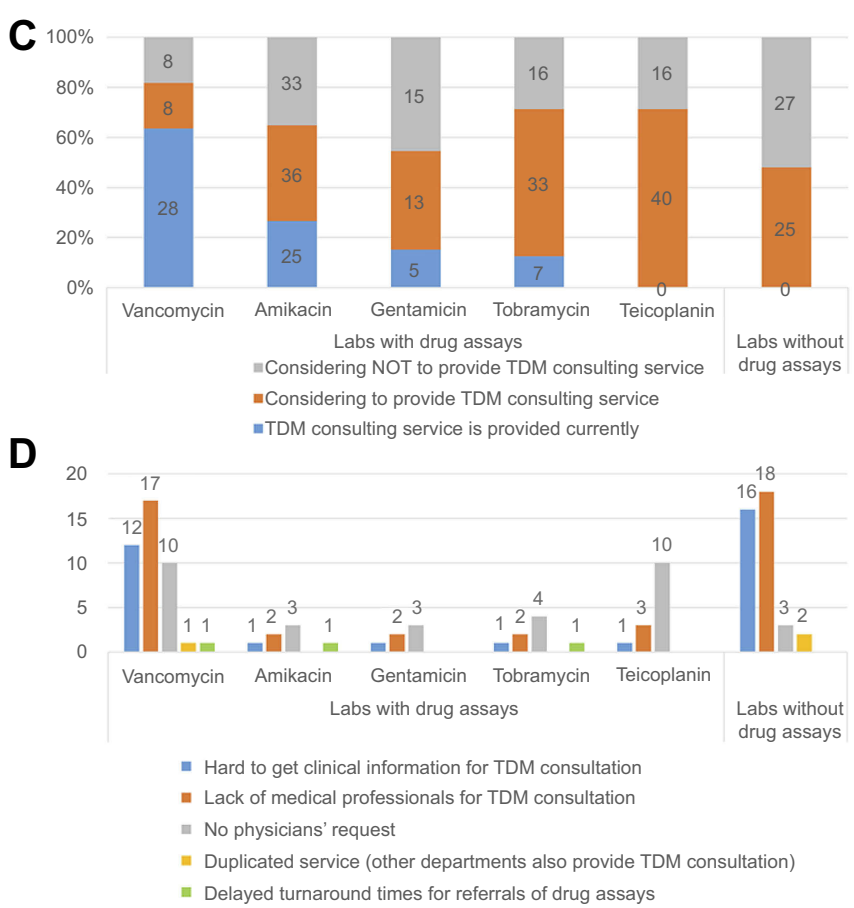

Figure I Utilization of drug assays by II 2 clinical laboratories in South Korea (number of respondents). (A) Whether laboratories provide drug assay services and have inhouse drug assay facilities for five antibiotics, (B) reasons for not having in-house drug assay facilities, (C) laboratories considering whether or not to provide therapeutic drug monitoring (TDM) consulting service in the future, and (D) reasons why therapeutic drug monitoring consulting services are not provided and/or will not be provided.

half of respondent laboratories expressed concern about the reliability of POC test results, but not smaller-scale devices (Figure 5). More than half of respondent laboratories answered that they expect to improve health care services by decreasing turnaround time, thereby increasing physician requests, and leading to additional clinical needs for TDM for other drugs.

\section{Discussion}

The results of this study provide information about the current status of TDM services, including drug assays and TDM consultation, in South Korea. We found that clinical laboratories in South Korea utilize drug assays for the five antibiotics studied, but only vancomycin TDM is widely used. Meanwhile, fewer laboratories are providing TDM consultations than drug assay results.

Laboratory directors in South Korea described their needs regarding drug assays and TDM consultation, as well as barriers to TDM utilization including operating costs, lack of human resources (medical professionals for TDM consultation), lack of user-friendly computer software and medical and laboratory information systems accessing patient information for TDM dose calculation, and reimbursement issues in South Korea. There are unmet needs for the improvement of drug assays including needs for new analytical methods, interpretation of results, consultation services, and QC. Furthermore, clinical laboratories provide various ranges of target concentrations for TDM for each antibiotic in this study. Even so, some laboratories do not provide information about target concentrations with drug assay results. One reason for the diversity of practice indicated in our survey results might differences in knowledge about TDM guidelines for different indications and dosages. ${ }^{3}$ If physicians are unable to interpret drug assay results in the context of corresponding target values, TDM consultation services may provide good guidance for physicians to improve patient care. ${ }^{4}$ Future studies and standardized guidelines for TDM for antibiotics pair with effective communication with physicians are needed to facilitate more appropriate utilization of TDM and improve patient health care. ${ }^{4}$

Clinical laboratories providing TDM consultation services use a variety of software packages. Several packages are available for dose calculations, some of which are expensive ( $\$ 1,520$ for a single seat license annually) and suited only for experienced users or medical professionals. $^{21}$ However, recent studies have reported that pharmacokinetics and pharmacodynamics vary among ethnicities. ${ }^{22}$ Future studies to obtain pharmacokinetic and pharmacodynamic information from Koreans 
Table 3 Current status of drug assays by 62 clinical laboratories with in-house drug assay facilities (numbers of responding laboratories)

\begin{tabular}{|c|c|c|c|c|c|}
\hline & Vancomycin & Amikacin & Gentamicin & Tobramycin & Teicoplanin \\
\hline \multicolumn{6}{|l|}{ Drug analysis operational hours } \\
\hline Working hours on workdays & 43 & 7 & 7 & 5 & 4 \\
\hline 24 hrs on workdays & 3 & & & & \\
\hline $24 / 7$ & 15 & 2 & 1 & 1 & \\
\hline Other (working hours on workdays and weekends) & 1 & & & & \\
\hline \multicolumn{6}{|l|}{ Frequency of drug analysis for a week } \\
\hline$<1$ day & & 1 & 1 & 1 & 1 \\
\hline I day & & & & & 1 \\
\hline 2 days & 2 & & & & I \\
\hline 3 days & 2 & & & & \\
\hline 4 days & 0 & & & & \\
\hline 5 days (workdays) & 35 & 5 & 5 & 4 & 1 \\
\hline 7 days (workdays and weekends) & 22 & 3 & 2 & 1 & \\
\hline Other (workdays upon request only) & 1 & & & & \\
\hline \multicolumn{6}{|l|}{ Frequency of IQC testing for drug assays } \\
\hline Every day, $\geq$ I time & 55 & 6 & 5 & 3 & 2 \\
\hline Other (< once per day) & 5 & 1 & 1 & 1 & 1 \\
\hline \multicolumn{6}{|l|}{ QC material for IQC } \\
\hline Commercialized QC materials & 62 & 6 & 5 & 3 & \\
\hline Others (in-house made) & & 1 & 1 & 1 & 3 \\
\hline \multicolumn{6}{|l|}{ Numbers of QC materials used for daily IQC } \\
\hline$<1$ & & 1 & 1 & I & 1 \\
\hline I & 2 & & & & \\
\hline 2 & 34 & 2 & 1 & 1 & 1 \\
\hline$\geq 3$ & 25 & 4 & 4 & 2 & 1 \\
\hline \multicolumn{6}{|l|}{ Participation in PT provided by K-EQAS } \\
\hline Yes & 61 & 6 & 5 & 4 & \\
\hline No & I & 2 & 2 & I & 4 \\
\hline \multicolumn{6}{|l|}{ Participation in PT provided by CAP } \\
\hline Yes & 15 & 5 & 4 & 3 & \\
\hline No & 47 & 4 & 4 & 3 & 5 \\
\hline
\end{tabular}

Abbreviations: CAP, College of American Pathologists; K-EQAS, Korean Association of External Quality Assessment Service; PT, proficiency test; IQC, internal quality control; TDM, therapeutic drug monitoring.

based on big data through increased utilization of TDM are needed to improve health care in Korea.

Recently, the WHO published an essential diagnostics list for laboratory testing in resource-limited settings and the list includes toxicology tests for therapeutic drug monitoring of amikacin, gentamicin, and vancomycin. ${ }^{23}$ In the present study, among 112 respondents, $27.7-36.6 \%$ of laboratories do not provide TDM for aminoglycoside and
$66.7 \%$ of laboratories do not provide TDM for teicoplanin. Furthermore, the teicoplanin drug assay is not standardized globally. A proficiency testing program for external quality assurance for the teicoplanin assay is not provided by the College of American Pathologists (CAP). In case of proficiency testing provided by LGC Standards, less than $1 \%$ of laboratories among 5,400 laboratories worldwide participate in the teicoplanin survey. This means that in most 
Table 4 Characteristics of drug assay results reporting and TDM consulting services by 62 clinical laboratories (both with and without in-house drug assay facilities, numbers of responding laboratories)

\begin{tabular}{|c|c|c|c|c|c|}
\hline & Vancomycin & Amikacin & Gentamicin & Tobramycin & Teicoplanin \\
\hline \multicolumn{6}{|c|}{ Average numbers of drug assay requests by physician per month } \\
\hline$<50$ & 7 & 41 & 45 & 39 & 19 \\
\hline$\geq 50$ and $<100$ & 14 & 2 & I & & 1 \\
\hline$\leq 100$ and $<300$ & 28 & 2 & & & \\
\hline$\leq 300$ and $<500$ & 8 & & & & \\
\hline$\geq 500$ & 5 & & & & \\
\hline \multicolumn{6}{|l|}{ Turnaround time for drug analysis } \\
\hline$<2$ hrs & 15 & 2 & I & 1 & 1 \\
\hline$\geq 2$ hrs and $<4$ hrs & 12 & 4 & 4 & 3 & \\
\hline$\geq 4 \mathrm{hrs}$ and $<24 \mathrm{hrs}$ & 32 & 3 & 3 & 2 & 2 \\
\hline$\geq 24 \mathrm{hrs}$ & 3 & 36 & 38 & 33 & 17 \\
\hline \multicolumn{6}{|c|}{ Institutions providing TDM consulting service to physicians } \\
\hline Yes & 36 & 10 & 10 & 7 & \\
\hline No & 26 & 9 & 8 & 10 & 16 \\
\hline TDM consulting service providers & & & & & \\
\hline Department of laboratory medicine & 20 & 7 & 7 & 6 & \\
\hline Department of clinical pharmacology & 7 & 3 & 3 & 2 & \\
\hline Department of Pharmacy & 21 & 5 & 5 & 3 & \\
\hline \multicolumn{6}{|c|}{ Average numbers of TDM consulting service requests by physicians per month } \\
\hline$<50$ & 16 & 8 & 10 & 7 & \\
\hline$\geq 50$ and $<100$ & 4 & 2 & & & \\
\hline$\geq 100$ and $<300$ & 10 & & & & \\
\hline$\geq 300$ and $<500$ & 2 & & & & \\
\hline$\geq 500$ & I & & & & \\
\hline \multicolumn{6}{|c|}{ Turnaround time for TDM consulting service } \\
\hline$<4 \mathrm{hrs}$ & I & & & & \\
\hline 4-24 hrs & 23 & 8 & 7 & 4 & \\
\hline$\geq 24 \mathrm{hrs}$ & 8 & & & & \\
\hline \multicolumn{6}{|c|}{ Computer software used for TDM consulting service } \\
\hline Simkin CAPCIL & 5 & I & I & & \\
\hline Abbott PKS & 22 & 8 & 7 & 6 & \\
\hline Mediware MwPharm & 3 & 2 & 3 & 1 & \\
\hline PKS \& Mwpharm (both two programs) & 2 & & & & \\
\hline Not available & 1 & & & & \\
\hline
\end{tabular}

Abbreviation: TDM, therapeutic drug monitoring.

parts of the word a 24/7 TDM service for this antibiotic is not readily available. Additional efforts to increase utilization of TDM for these antibiotics are needed to achieve high-quality laboratory diagnostic results for clinical laboratories in South Korea. Considering the fact that TDM for antibiotics is an indispensable part of appropriate treatment and management of various infectious diseases, national guidelines for TDM practices to provide appropriate care for patients are needed in South Korea.

Laboratory directors of clinical laboratories in South Korea indicated that there are needs for other platformbased drug assays, with the most common reason being turnaround time for POC test devices and high operating costs for smaller-scale platform devices. This finding 
A

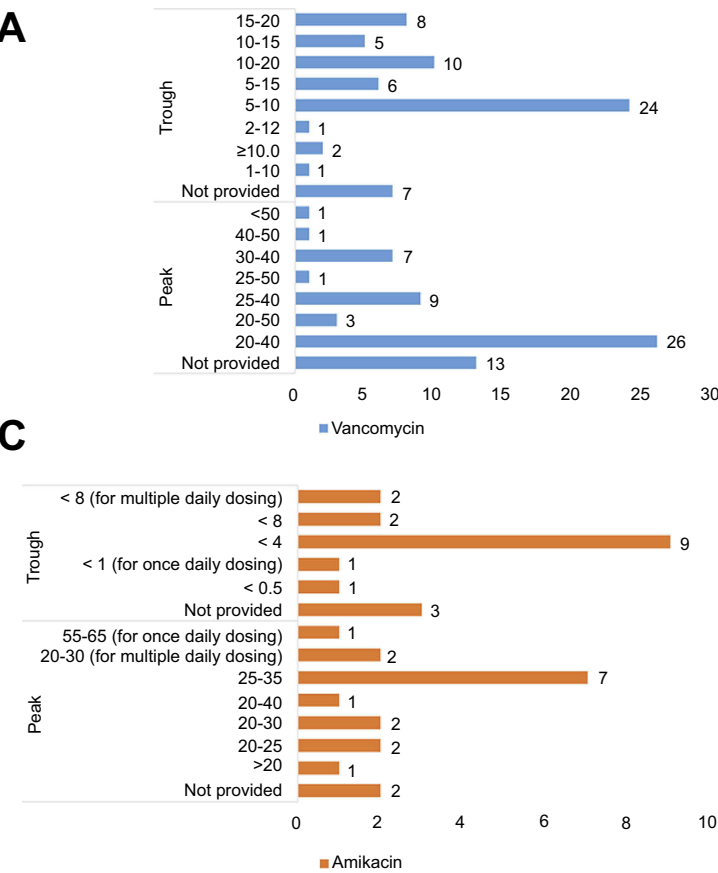

B

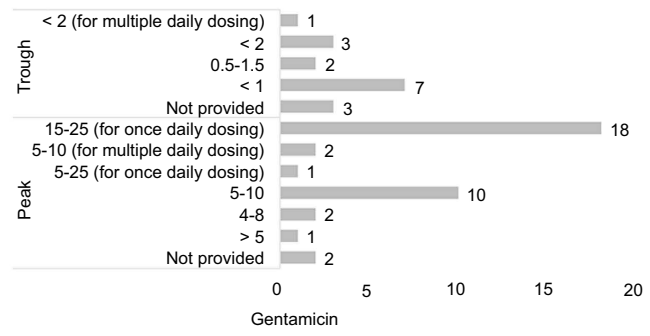

D

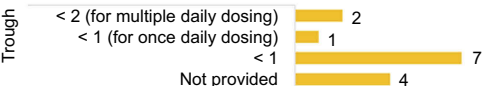

Not provided $15-25$ (for

$15-25$ (for once daily dosing)
$5-10$ (for multiple daily dosing)

ङ
$\Phi$$\quad \begin{array}{r}5 \text { (for multiple daily dosing) } \\ \text { Not provided }\end{array} \quad 1$

0
- Tobramycin

E

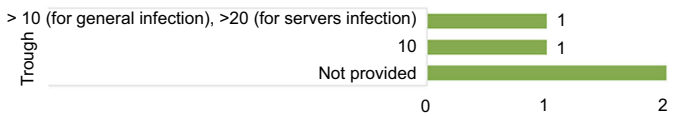

- Teicoplanin

Figure 2 Target concentrations for therapeutic drug monitoring of five antibiotics provided by clinical laboratories to physicians: (A) vancomycin, (B) amikacin, (C) gentamicin, (D) tobramycin, and (E) teicoplanin. The Y-axis represents target concentration (ug/mL). The X-axis represents number of respondents (laboratories). No clinical laboratories provided target peak concentrations for teicoplanin.

suggests that these are the most critical factors to help increase use of such instrument types for drug assays. Developers should focus on these factors to approach and develop new assay platforms to increase test utilization. In this study, more than half of respondent laboratories expressed concern about the reliability of POC test results, but not smaller-scale devices. To maximize the success probability of POC tests, a substantial amount of attention should
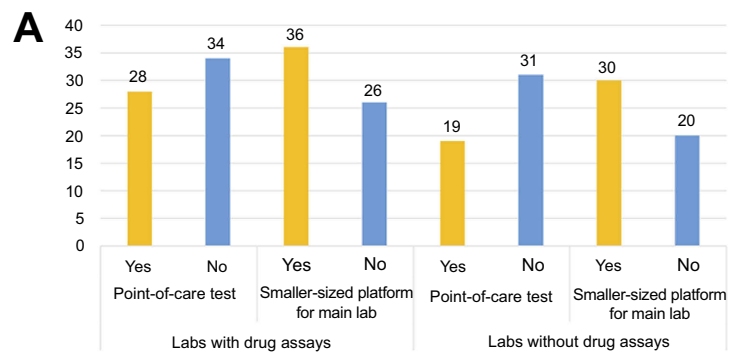

Labs with drug assays

Labs without drug assays

\section{C}

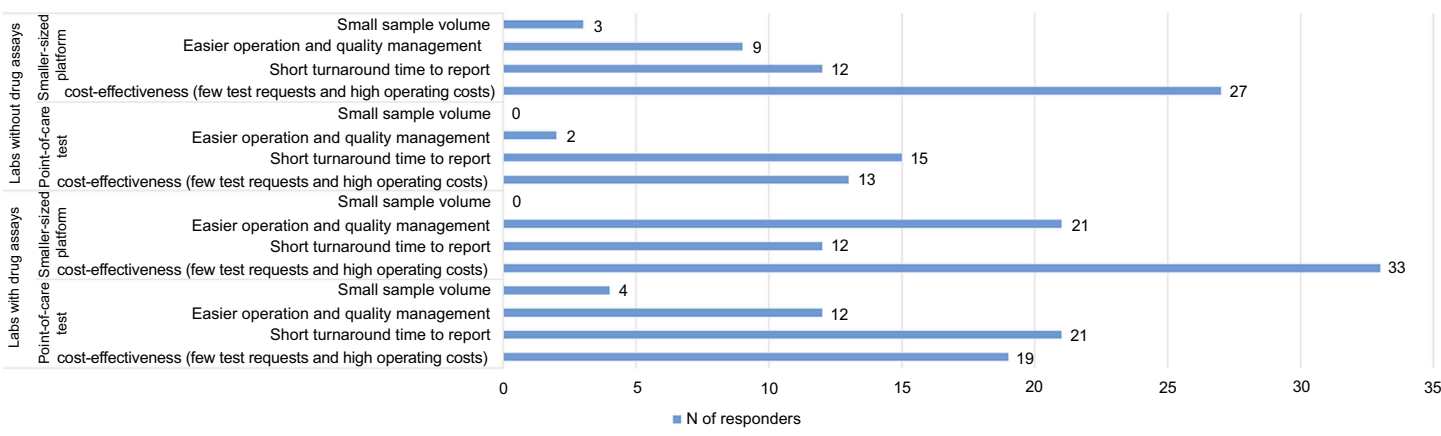

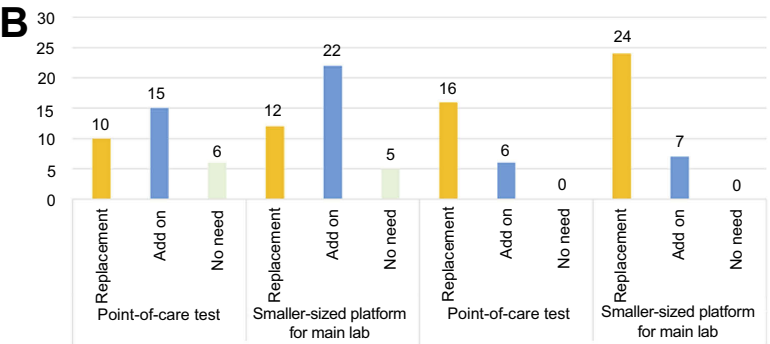

Labs with drug assays Labs without drug assays

Figure 3 Expectations for other platform-based drug assays, such as point-of-care or smaller scale devices (number of respondents). (A) Needs for other platform-based drug assays, (B) consideration of future installation of other platform-based drug assays, and (C) reasons for needs expressed regarding other platform-based drug assays. 

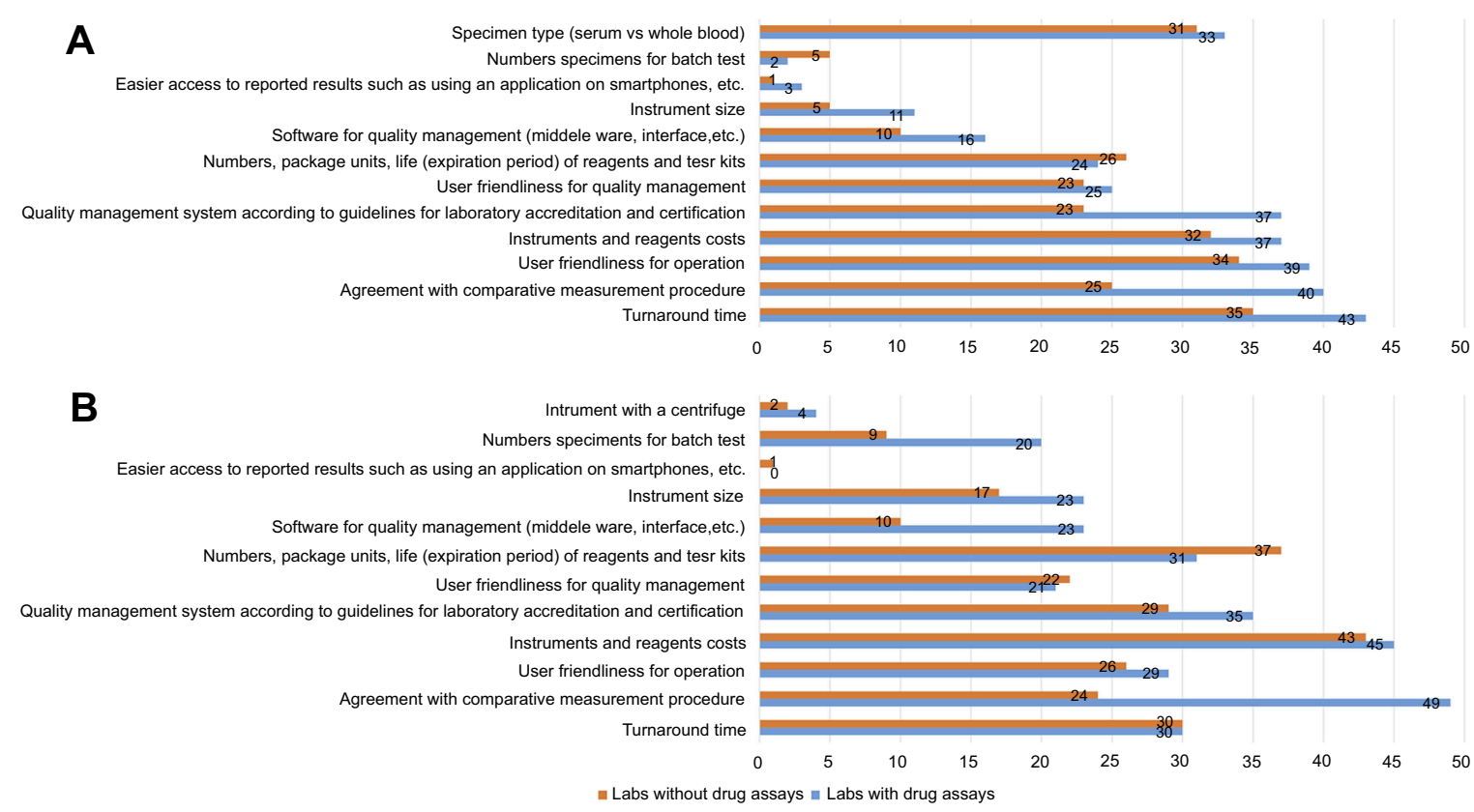

Figure 4 Expectations of the requirements for using other platform-based drug assays, such as (A) point-of-care and (B) smaller scale devices.

A

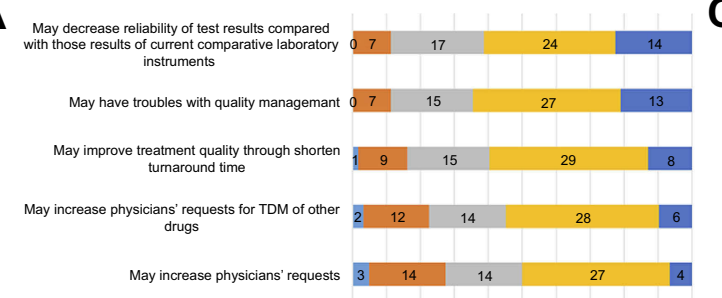

$0 \%$ 10\% $20 \%$ 30\% $40 \%$ 50\% $60 \%$ 70\% $80 \% 90 \% 100 \%$

B

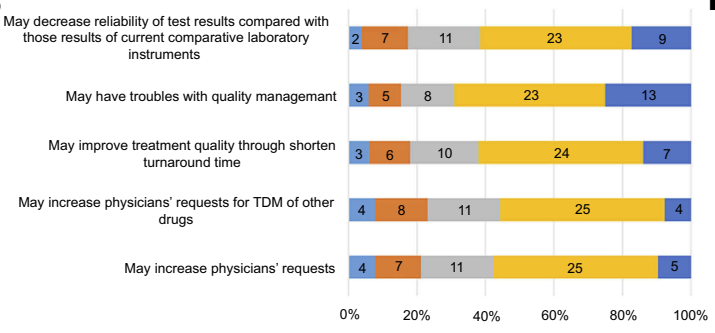

| Strongly disagree = Somewhat disagree $=$ Neither agree or disagree $=$ Somewhat agree | Strongly agree
C

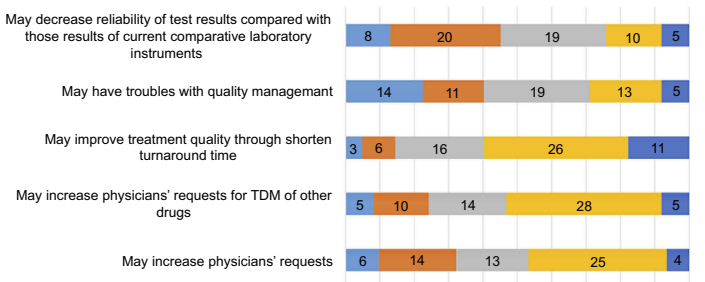

$0 \% 10 \% 20 \% 30 \% 40 \% 50 \%$ 60\% $70 \%$ 80\% $90 \% 100 \%$

D

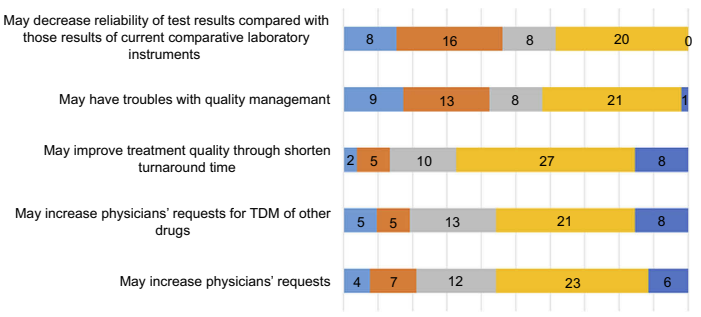

$0 \% 10 \%$ 20\% $30 \%$ 40\% 50\% $60 \%$ 70\% $80 \%$ 90\% $100 \%$

=Strongly disagree $\|$ Somewhat disagree $=$ Neither agree or disagree $=$ Somewhat agree $\|$ Strongly agree

Figure 5 Concerns of the impacts of other platform-based drug assays. (A) Responses from laboratories with in-house drug assays (concerns of the impacts of point-of-care tests), (B) responses from laboratories with in-house drug assays (concerns of the impacts of smaller scale devices), (C) responses from laboratories without in-house drug assays (concerns of the impacts of point-of-care tests), and (D) responses from laboratories without in-house drug assays (concerns of the impacts of smaller scale devices).

be devoted to clinical validation and implementation strategies beyond the initial technology proof of concept. ${ }^{24}$

The strength of this study is the inclusion of nationwide data from clinical laboratories across various practice settings in South Korea. It is of note that $91.2 \%(62 / 68)$ of clinical laboratories with in-house drug assay facilities participated in our survey. The overall response rate of clinical laboratories without in-house drug assay facilities was low $(29.9 \%, 50 / 167$ laboratories), which is an unfortunate limitation inherent to all survey-based studies. Another limitation is the potential selection bias toward laboratory directors with an interest in drug assays and TDM consultation. 
Furthermore, we sent the survey to members of the KSLM, and as a result, responses were limited to clinical pathologists who practice in South Korea. Therefore, the results of our study may not be generalizable to physicians outside South Korea, where insurance policies and drug assay and TDM consulting service availability and costs differ. ${ }^{14}$ However, this study still has valuable information about TDM practice in a country with different resource settings. To the best of our knowledge, this is the first study to provide detailed and comprehensive information about TDM practice and utilization of TDM services including both TDM drug assays and TDM consultation as well as the professional perspectives of point-of-care testing in TDM practice.

\section{Conclusion}

In this study we showed that clinical laboratories in South Korea are already utilizing drug assays, but relatively few provide TDM consultation. Although laboratory directors recognize the need for drug assays and TDM consultation, major barriers such as high operating costs, time lag from test to results, and lack of overall knowledge about TDM, persist. Removing these barriers could increase the use of TDM, both among clinical laboratories already using TDM and clinical laboratories that do not use TDM.

\section{Acknowledgments}

We thank all of our colleagues who participated in this survey. This article is based upon work supported by the Ministry of Trade, Industry \& Energy (MOTIE, Korea) under Industrial Technology Innovation Program. No.10080648,'Antibiotics monitoring point-of-care test.

\section{Disclosure}

The authors report no conflicts of interest in this work.

\section{References}

1. van Altena R, Dijkstra JA, van der Meer ME, et al. Reduced chance of hearing loss associated with therapeutic drug monitoring of aminoglycosides in the treatment of multidrug-resistant tuberculosis. Antimicrob Agents Chemother. 2017;61(3). doi:10.1128/AAC.01400-16

2. World Health Organization. 2016-2017. Global antimicrobial resistance surveillance system (GLASS) report: early implementation 2017. Available from: https://apps.who.int/iris/bitstream/han dle/10665/259744/9789241513449-eng.pdf;jsessionid= 0BD793C9FADB1F9B559EA9E8C6102E63? sequence=1. March 14, 2019.

3. Reeves D, Lovering A, Thomson A. Therapeutic drug monitoring in the past 40 years of the journal of antimicrobial chemotherapy. $J$ Antimicrob Chemother. 2016;71(12):3330-3332. doi:10.1093/jac/dkw408

4. Bates DW, Soldin SJ, Rainey PM, Micelli JN. Strategies for physician education in therapeutic drug monitoring. Clin Chem. 1998;44(2):401407.
5. Ye ZK, Tang HL, Zhai SD. Benefits of therapeutic drug monitoring of vancomycin: a systematic review and meta-analysis. PLoS One. 2013;8(10):e77169. doi:10.1371/journal.pone.0077169

6. Schroeder LF, Guarner J, Amukele TK. Essential diagnostics for the use of world health organization essential medicines. Clin Chem. 2018;64(8):1148-1157. doi:10.1373/clinchem.2017.275339

7. Filippone EJ, Kraft WK, Farber JL. The nephrotoxicity of vancomycin. Clin Pharmacol Ther. 2017;102(3):459-469. doi:10.1002/cpt.726

8. Murray BE, Arias CA, Nannini EC. Glycopeptides (Vancomycin and Teicoplanin), Streptogramins (Quinupristin-Dalfopristin), Lipopeptides (Daptomycin), and Lipoglycopeptides (Telavancin). In: Mandell, Douglas, and Bennett's Principles and Practice of Infectious Diseases (Eighth Edition). Elsevier Health Sciences, Philadelphia; 2015: 377-400.e374.

9. Craig WA. Optimizing aminoglycoside use. Crit Care Clin. 2011;27 (1):107-121. doi:10.1016/j.ccc.2010.11.006

10. Banerjee S, Narayanan M, Gould K. Monitoring aminoglycoside level. BMJ. 2012;345:e6354.

11. Huth ME, Han KH, Sotoudeh K, et al. Designer aminoglycosides prevent cochlear hair cell loss and hearing loss. J Clin Invest. 2015;125(2):583-592. doi:10.1172/JCI77424

12. Chae H, Lee JJ, Cha K, et al. Measurement of teicoplanin concentration with liquid chromatography-tandem mass spectrometry method demonstrates the usefulness of therapeutic drug monitoring in hematologic patient populations. Ther Drug Monit. 2018;40(3):330-336. doi:10.1097/FTD.0000000000000498

13. Ko DH, Gu GG, Cho EJ, Shin ES, Chun S, Kim JH. As therapeutic drug monitoring subcommittee, Korean association of external quality assessment service. Annual report on the external quality assessment of therapeutic drug monitoring and testing for drugs of abuse in Korea (2015). J Lab Med Qual Assur. 2016;38(1):11-21. doi:10.15263/jlmqa.2016.38.1.11

14. Ab Rahman AF, Ahmed Abdelrahim HE, Mohamed Ibrahim MI. A survey of therapeutic drug monitoring services in Malaysia. Saudi Pharm J. 2013;21(1):19-24. doi:10.1016/j.jsps.2012.01.002

15. Grossberg LB, Papamichael K, Feuerstein JD, Siegel CA, Ullman TA, Cheifetz AS. A survey study of gastroenterologists' attitudes and barriers toward therapeutic drug monitoring of Anti-TNF therapy in inflammatory bowel disease. Inflamm Bowel Dis. 2017;24(1):191197. doi:10.1093/ibd/izx023

16. Guo W, Guo GX, Sun C, et al. Therapeutic drug monitoring of psychotropic drugs in China: a nationwide survey. Ther Drug Monit. 2013;35(6):816-822. doi:10.1097/FTD.0b013 e318296a2ff

17. Norris RL, Martin JH, Thompson E, et al. Current status of therapeutic drug monitoring in Australia and New Zealand: a need for improved assay evaluation, best practice guidelines, and professional development. Ther Drug Monit. 2010;32(5):615-623. doi:10.1097/ FTD.0b013e3181ea3e8a

18. Tabah A, De Waele J, Lipman J, et al. The ADMIN-ICU survey: a survey on antimicrobial dosing and monitoring in ICUs. J Antimicrob Chemother. 2015;70(9):2671-2677. doi:10.1093/jac/dkv165

19. Charmillon A, Novy E, Agrinier N, et al. The ANTIBIOPERF study: a nationwide cross-sectional survey about practices for beta-lactam administration and therapeutic drug monitoring among critically ill patients in France. Clin Microbiol Infect. 2016;22(7):625-631. doi:10.1016/j.cmi.2016.04.019

20. Cotta MO, Dulhunty JM, Roberts JA, Myburgh J, Lipman J. Should beta-lactam antibiotics be administered by continuous infusion in critically ill patients? A survey of Australia and New Zealand intensive care unit doctors and pharmacists. Int $J$ Antimicrob Agents. 2016;47(6):436-438. doi:10.1016/j. ijantimicag.2016.02.017

21. Fuchs A, Csajka C, Thoma Y, Buclin T, Widmer N. Benchmarking therapeutic drug monitoring software: a review of available computer tools. Clin Pharmacokinet. 2013;52(1):9-22. doi:10.1007/s40262012-0020-y 
22. Baietto L, Corcione S, Pacini G, Perri GD, D'Avolio A, De Rosa FG. A 30-years review on pharmacokinetics of antibiotics: is the right time for pharmacogenetics? Curr Drug Metab. 2014;15(6):581-598.

23. World Health Organization. The 20th WHO Essential Medicines List (EML) and the 6th WHO Essential Medicines List for Children (EMLc) updated in March 2017. Available from: https://www.who.int/medi cines/publications/essentialmedicines/en/. Accessed March 14, 2019.
24. Wang P, Kricka LJ. Current and emerging trends in point-of-care technology and strategies for clinical validation and implementation. Clin Chem. 2018;64(10):1439-1452. doi:10.1373/clinchem.2018.287052

\section{Publish your work in this journal}

Infection and Drug Resistance is an international, peer-reviewed openaccess journal that focuses on the optimal treatment of infection (bacterial, fungal and viral) and the development and institution of preventive strategies to minimize the development and spread of resistance. The journal is specifically concerned with the epidemiology of antibiotic resistance and the mechanisms of resistance development and diffusion in both hospitals and the community. The manuscript management system is completely online and includes a very quick and fair peerreview system, which is all easy to use. Visit http://www.dovepress.com/ testimonials.php to read real quotes from published authors. 